\title{
Implementation of Packaged Integrated Antenna With Embedded Front End for Bluetooth Applications
}

\author{
Mihai Rotaru, Member, IEEE, Lim Ying Ying, Member, IEEE, Haridas Kuruveettil, Yang Rui, \\ Alexander P. Popov, Senior Member, IEEE, and Chua Chee-Parng
}

\begin{abstract}
The design, integration and realization of system in enhanced package approach towards fully functional system level integration by using a compact Bluetooth USB dongle as the demonstrator is presented here. The integration was done on FR4 substrates, which is totally compatible with today's printed circuit board manufacturing capability. A commercially available Bluetooth integrated chip was chosen as the chipset of our demonstrator, and a package integrated antenna together with an embedded front end completes the system in package integration. The front end developed here is based on an embedded meander line combline filter and an embedded transformer balun. The filter has a $35 \%$ area reduction when compared with the classical combline filter and similar performance. The balun has the coils distributed on three layers that minimized the board area needed it and optimizes the performances. The proposed packaged integrated antenna approach is successfully demonstrated here and the new module shows excellent performance when compared with a commercial solution, surpassing the normal Bluetooth class II dongle range which is up to $10 \mathrm{~m}$ and increasing the module range up to $120 \mathrm{~m}$ without an extra power amplifier.
\end{abstract}

Index Terms-Dielectric resonator antenna (DRA), embedded front end, embedded passives, package integrated antenna, system in package $(\mathrm{SiP})$, wireless module.

\section{INTRODUCTION}

$\mathbf{R}$ ADIO-FREQUENCY (RF) systems have progressed remarkably in the last ten years mostly due to the popularization of cellular phone handsets and other hand held devices that require wireless communication. It is expected that this trend will continue in the coming years as new applications that require wireless communication start to appear. New applications like logistics and intelligent home networks medical information systems are still in emerging phase but have huge potential market. The advancements in RF silicon and module in-

Manuscript received March 28, 2007; revised February 20, 2008. Published August 6, 2008 (projected). This work was recommended for publication by Associate Editor L.-T. Hwang upon evaluation of the reviewers comments.

M. Rotaru was with the Institute of Microelectronics (IME), 116785 Singapore. He is now with School of Electronics and Computer Science, University of Southampton, SO17 1BJ Southampton, U.K. (e-mail: mr@ecs.soton.ac.uk).

Y. Y. Lim and K. Haridas are with the Institute of Microelectronics (IME), 116785, Singapore (e-mail: limyy@ime.a-star.edu.sg; kharidas@ime.a-star. edu.sg).

Y. Rui was with the Institute of Microelectronics (IME), 116785 Singapore. He is now with Huawei Technology Company, Ltd., Shenzhen 518129, China.

A. P. Popov is with the Department of Electrical and Computer Engineering, National University of Singapore, 119260 Singapore.

C. Chee-Parng is with the Perlos Asia Pte Ltd., Nordic European Centre, 609927 Singapore.

Color versions of one or more of the figures in this paper are available online at http://ieeexplore.ieee.org.

Digital Object Identifier 10.1109/TADVP.2008.927840

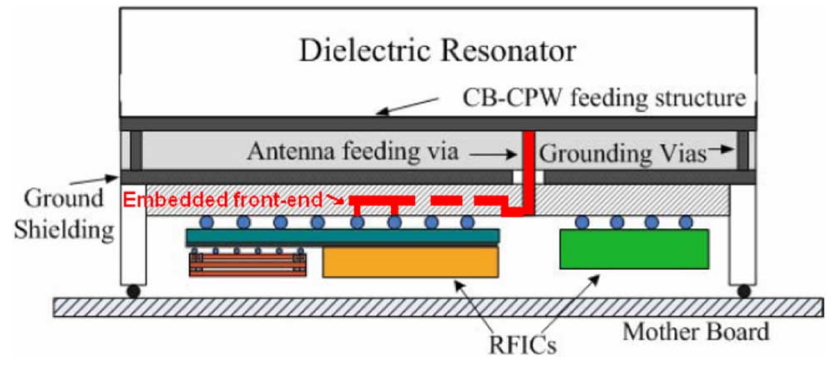

Fig. 1. Concept of the wireless module using the package integrated antenna approach.

tegration are expected to enable and support the frenzy wireless market development which is expected to grow with $20 \%-100 \%$ per year in the next several years [1].

To meet a variety of requirements on RF modules such as technical performance, size, and cost considerations, two major design concepts system in package (Sip) and system on package (SoP) were introduced recently [2]. The difference between these two concepts is that in SoP approach some components of the system may be embedded in the package, while in SiP approach some components of the system maybe embedded in the substrate. However, the basic functions of the packages, namely, protection from hazardous environment, thermal protection, and electrical connection with the rest of the circuitry, remain the same for the both design concepts.

In the present paper, a novel system in enhanced package (SieP) design concept is introduced and demonstrated. The core of this design concept is using a package with enhanced functions that acts as a transducer providing additional connectivity between the system and the environment. In particular, in the RF module design presented in the paper the module package is designed to operate as a dielectric resonator antenna (DRA), while the antenna feed is integrated with the rest of the circuitry on a low loss substrate (Fig. 1).

The main advantage of this approach is that the maximum available space is provided for the radiating element without significant increase of the module size. The shape of a dielectric resonator package and a particular feed structure is chosen to provide a specified circular polarized radiation pattern and the specified bandwidth of operation.

The proposed paper presents the design, integration, and realization of SieP approach towards fully functional system level integration by using a compact Bluetooth USB dongle as the demonstrator. The integration was done on FR4 substrates, which is totally compatible with today's printed circuit board (PCB) manufacturing capability. Further miniaturization and 
hybrid integrations still can be pursued by introducing more costly fabrication process but it has been avoided in this work as we try to stay as close as possible to the simple and well known FR4-PCB process.

The commercially available CSR BC04-ROM chip [4], [5] was chosen as the main chipset of our demonstrator, which is a system-on-chip (SoC) solution for Bluetooth application having integrated at chip level the radio and baseband together with $4 \mathrm{MB}$ of memory. Here we have concentrated on system level design, integration and functionality verification. The whole RF front end was implemented by using an embedded band pass filter (BPF) and embedded Balun in PCB [7].

The embedded front end used in this module includes a meander line combline filter that has $35 \%$ area reduction when compared with the classical combline filter and it has similar performance-better that $-2.5 \mathrm{~dB}$ loss in the pass band, $110-\mathrm{MHz}$ bandwidth and better than $-15 \mathrm{~dB}$ rejection outside of the pass band. The front end also includes an embedded transformer Balun, with the primary and secondary (differential) coils distributed over three layers. Due to its 3-D structure the Balun occupies a smaller space than the regular planar Baluns using the same PCB technology. The measured data of the Balun showed that the structure has a wide bandwidth of about $180 \mathrm{MHz}$ for a better than $-0.9 \mathrm{~dB}$ differential loss. Also the amplitude imbalance of the device has been found to be acceptable.

Following the introductory Section I, Section II of this paper concentrates on the antenna characteristics and design. Further in Section III the front end of the module is presented. Detailed description of the design, modeling, and characterization of the embedded filter and balun are given. Section IV is dedicated to the system realization using the technologies described in Sections II and III. Section V shows the functional testing results where the new functional Bluetooth module is compared with existent modules in terms of distance and speed. Finally in Section VI we conclude the works through some key thoughts.

\section{ANTENNA CHARACTERISTICS AND DESIGN}

\section{A. Package Integrated Antenna}

As explained in Section I SiP offers an elegant and cost effective solution for a compact functional system that needs integration of few technologies. For example, a wireless handheld module transmitting and receiving information, needs an effective antenna. In our proposed solution SieP, an enhanced module is obtained by optimizing the space/efficiency ratio of the radiating element. To achieve this a dielectric resonator antenna is proposed and integrated at package level, which maximizes the use of the available space without compromising the size of the module. Most of the available published solution [3], [6], [10]-[17], are based on planar antenna structures that suffer from low efficiency, reduce bandwidth, and are very prone to detuning. Our previous work [7]-[9] has introduced the concept of package integrated dielectric resonator. Here, the same structure is used (Fig. 1), with the necessary modification such it covers the Bluetooth band. The antenna structure is shown in Figs. 2 and 3, it comprises in a cylindrical dielectric resonator and an antenna feed, that are designed on one side of the sub-

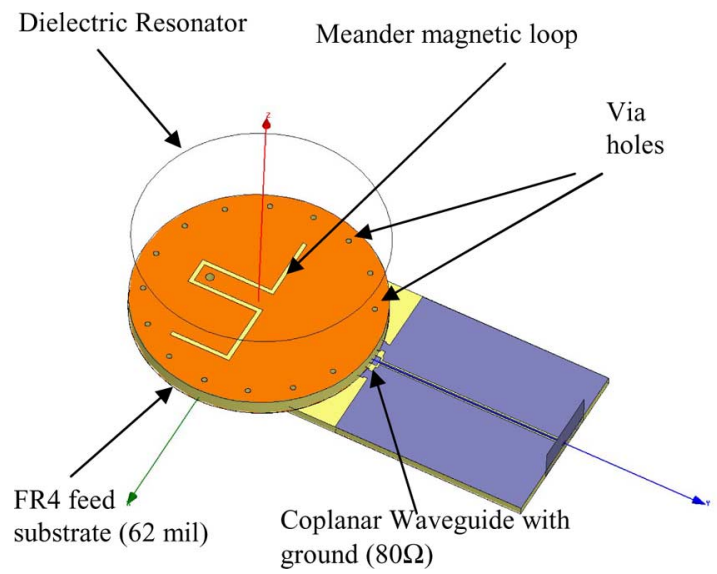

Fig. 2. Top view of the integrated antenna.

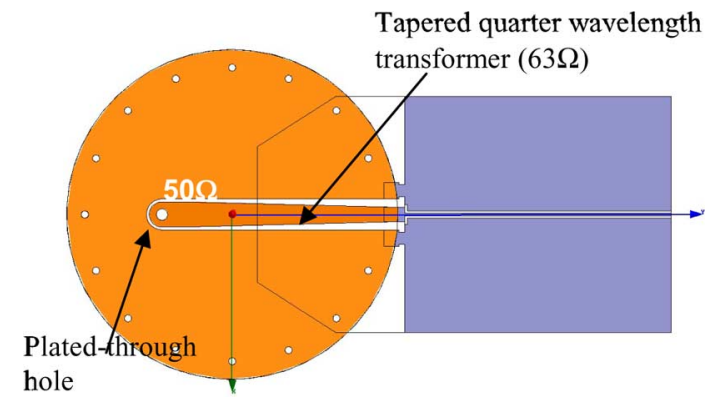

Fig. 3. Bottom view of the integrated antenan.

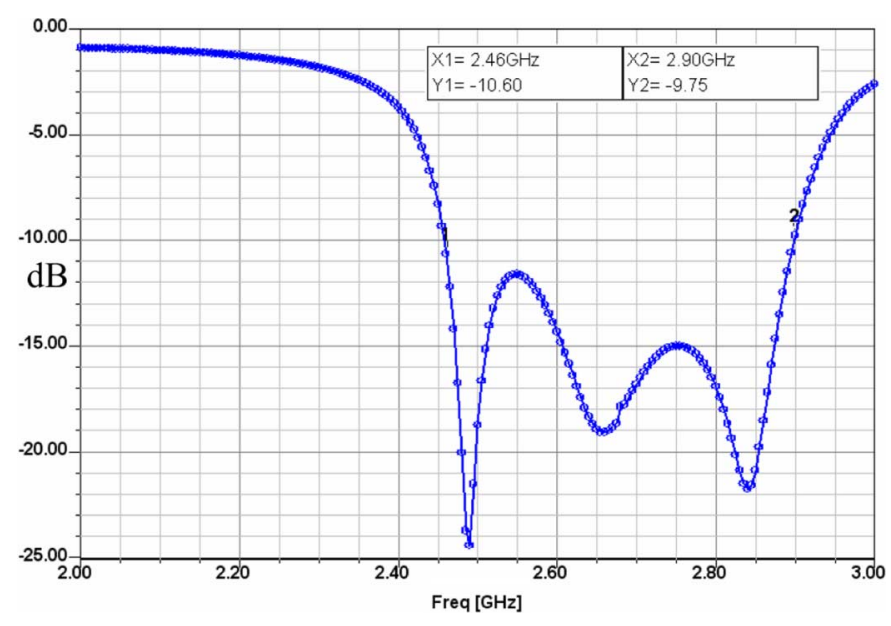

Fig. 4. Simulated return loss.

strate. The active devices are mounted on the other side of the substrate, and the power and ground system is designed in such a way that can provide appropriate power to the active devices and shielding them from the radiator. (Fig. 1).

The resonator is excited trough a meander magnetic dipole (Fig. 2) that ensures a circular polarization for the antenna. A via hole connects the top layer to a $50-\Omega$ coplanar waveguide (CPW) on the bottom layer. Subsequently, the $50-\Omega$ CPW is matched to the Bluetooth module test-board which operates in an $80-\Omega$ environment, using a tapered quarter-wavelength transformer $(63 \Omega)$. Via holes are used to provide grounding from the top conductor to the bottom ground. 


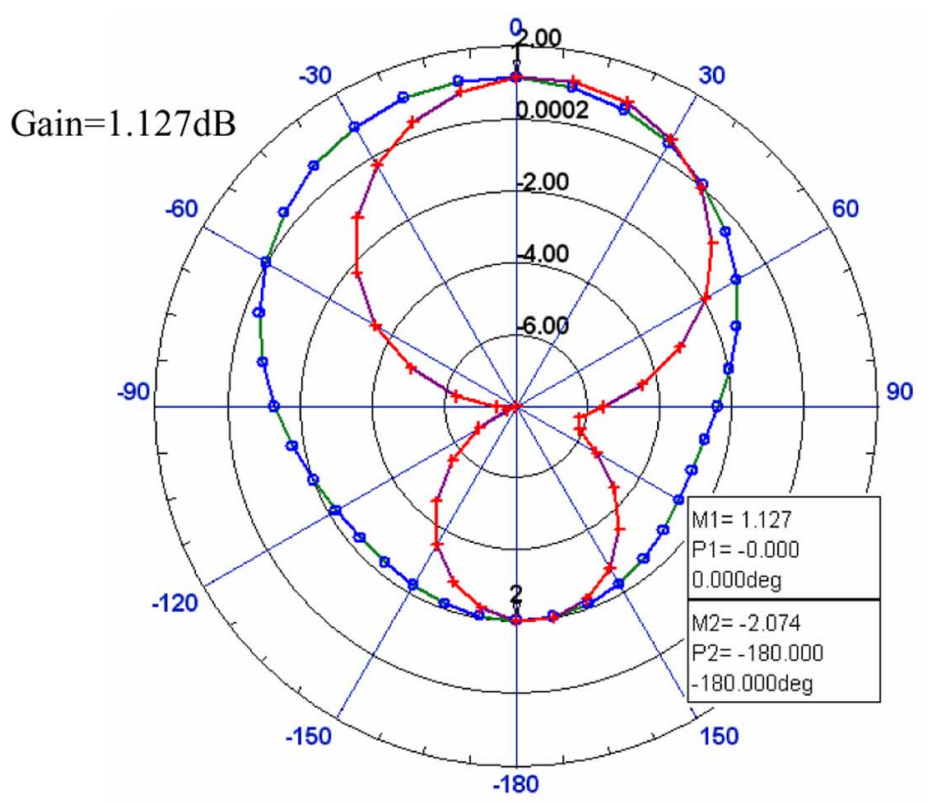

(a)

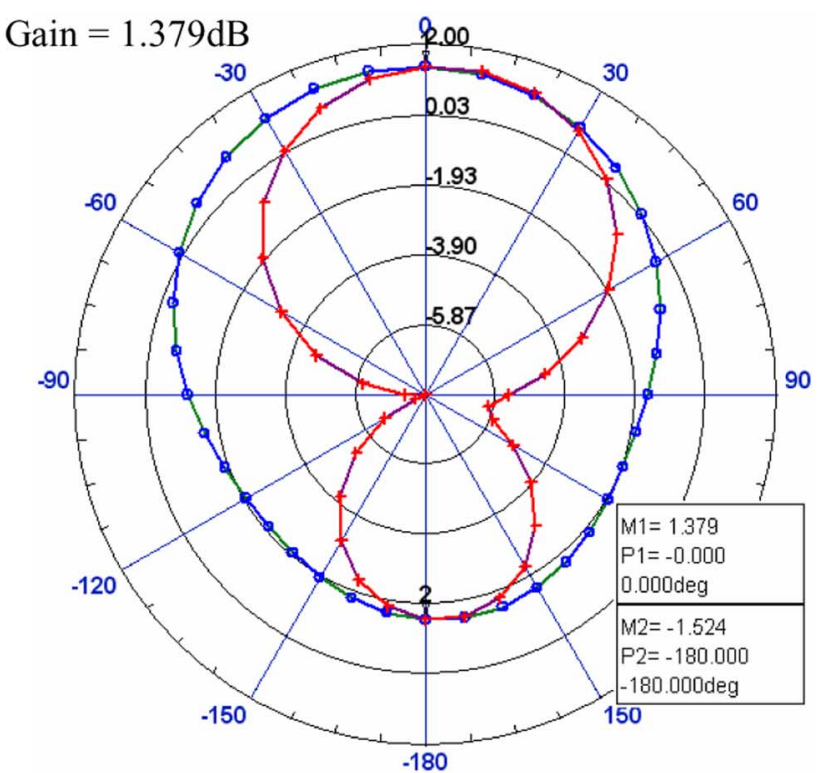

(b)

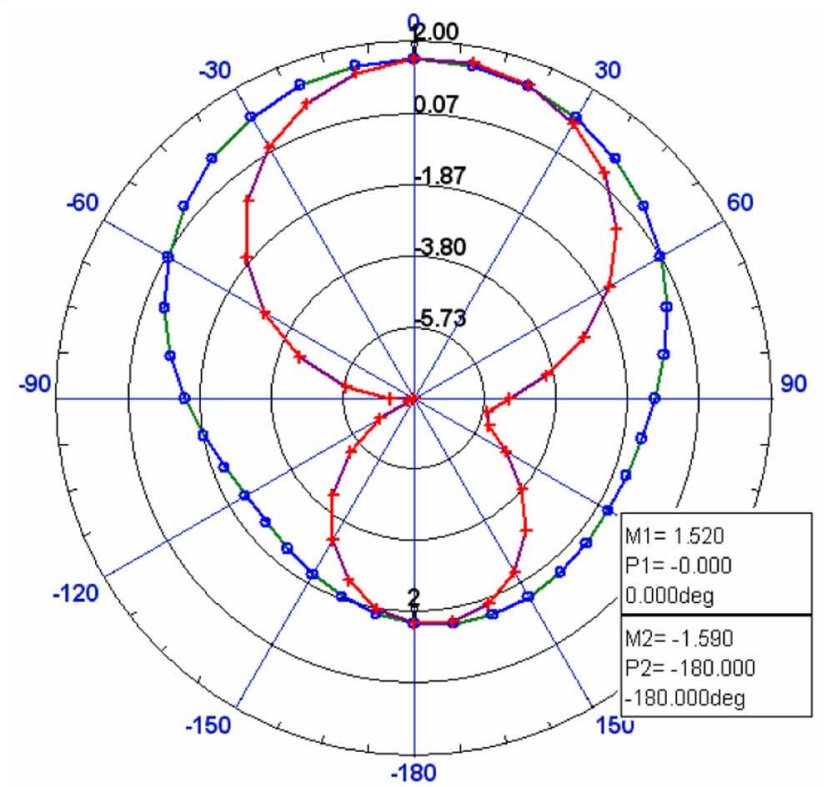

(c)

Fig. 5. Antenna radiation pattern at (a) $2.46 \mathrm{GHz}$, (b) $2.48 \mathrm{GHz}$, and (c) 2.50 .

To give sufficiently good grounding as a PEC sidewalls simulated in previous designs, the antenna structure is modeled with increasing number of via holes until the scattering parameter $S_{11}$ are almost identical to the results obtained using PEC sidewalls. The minimum number of via holes obtained through this method is found to be 15 equally spaced vias. The parameters of the simulated optimized antenna are presented in Figs. 4 and 5. The return loss (Fig. 4) shows three resonances at 2.50, 2.65, and $2.85 \mathrm{GHz}$. The antenna has a $16 \%$ impedance bandwidth at a level of $-10 \mathrm{~dB}$ covering well beyond the $100 \mathrm{-MHz}$ bandwidth necessary for the Bluetooth application.

A quick look at the radiation patterns at the frequencies of $2.46,2.48$, and $2.50 \mathrm{GHz}$ shows the antenna having a smooth and symmetrical radiation pattern in the broadside direction within the wide bandwidth of $0.44 \mathrm{GHz}$.

\section{Front END DESIGN AND CharacteraZATION}

The front end circuitry between the antenna and the differential input of the RF chip comprises of an impedance matching network, a band pass filter (BPF), a balun, and another matching network.

\section{A. Embedded Combline Filter}

One of the most important components of the front end circuitry is the band pass filter (BPF). For the Bluetooth applications, the filter has to satisfy specification of a low insertion 


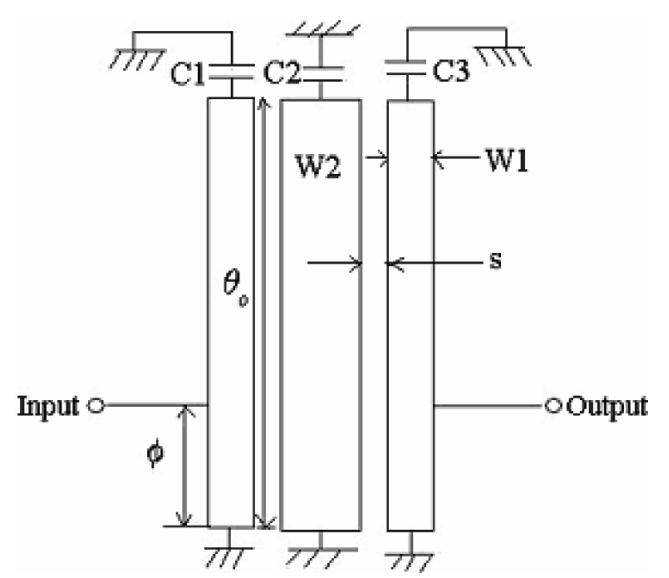

Fig. 6. Initial combline filter.

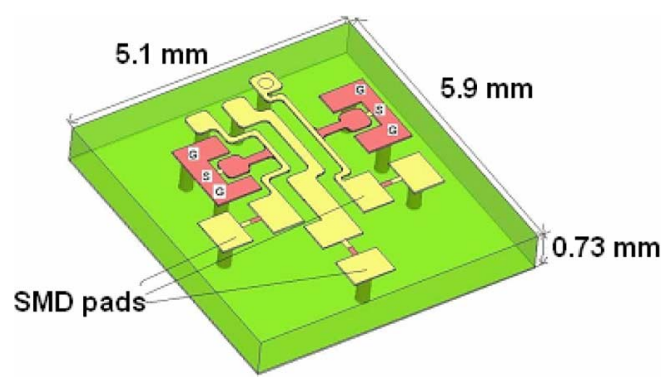

Fig. 7. Meander combline filter-3-D view.

loss $(\leq-2.5 \mathrm{~dB})$ over a narrow bandwidth of $100 \mathrm{MHz}$, center around $2.45 \mathrm{GHz}$ in the industrial, scientific, and medical (ISM) band. Ceramic filters are normally used in this type of configuration but here a combline filter is embedded within the substrate of the module. The area occupied by the filter is similar with a normal ceramic counterpart but it comes for free as is part of the board fabrication cost. Combline filters are popular choice due to their good performances and a relative compact size. To further reduce the size a novel design based on a meander line configuration is used here [19]. Combline filter structures are constructed from mutual coupled resonators, here three resonators are used. Each resonator is built from a shunt shorted transmission lines loaded with a capacitance, which reduces the length of the transmission line. Electrically at the center frequency of the filter each resonator appears as $90^{\circ}$ long. The coupling between neighboring resonators is done through a combination of magnetic and electric fields existent around each of the short transmission lines.

Microstrip on FR4 substrate transmission lines are used here. The design follows the methodology described in [18]. The initial structure (Fig. 6) has two different widths for the microstrips, W1, W2 which are 100 and $200 \mu \mathrm{m}$, respectively. The loading capacitance for the two different microstrips is $1.5 \mathrm{pF}(\mathrm{C} 1, \mathrm{C} 3)$ for outer microstrips and $0.47 \mathrm{pF}(\mathrm{C} 2)$ for the middle transmission line. The spacing between resonators was $280 \mu \mathrm{m}$ and the length of the lines was $3.71 \mathrm{~mm}$ which yields a resonance at $2.40 \mathrm{GHz}$. A good matching to $50 \Omega$ has been ensured by choosing an appropriate tapping distance $\phi$ from the grounding point. The area occupied by the filter was minimized

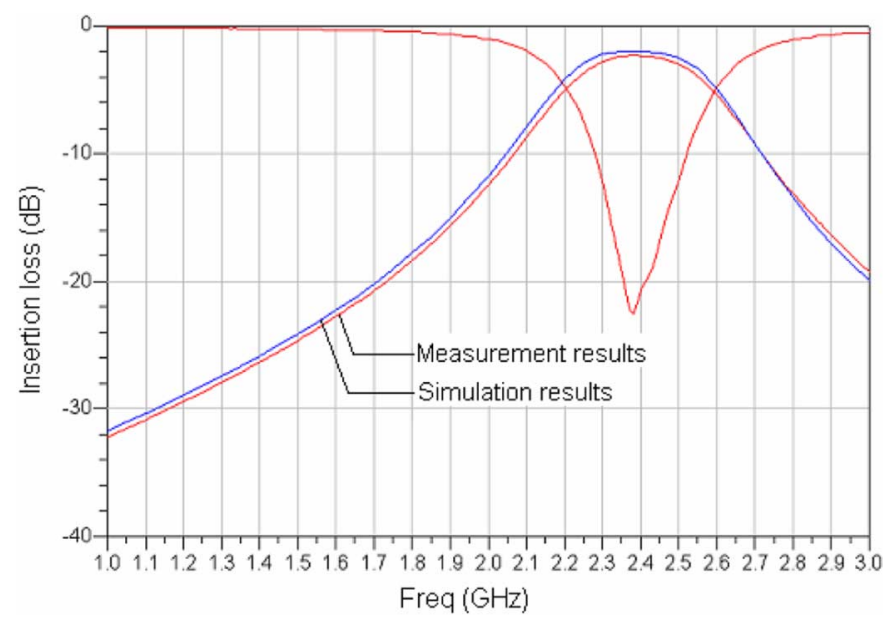

Fig. 8. Insertion and return loss for the combline filter-measured and simulated results.

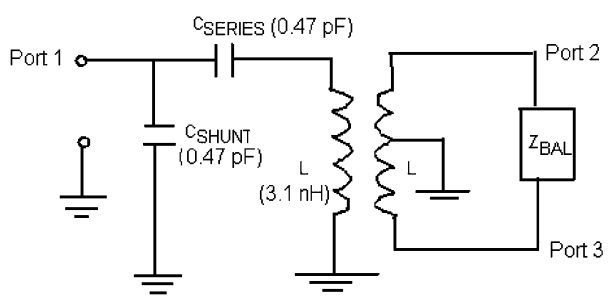

Fig. 9. Equivalent circuit of the balun and its matching network.

further through introduction of bends in the microstrip lines. All the other parameters, width, spacing, tapping position were kept fixed. The resultant structure had a $35 \%$ reduction in area (Fig. 7). The new structure was then solved and optimized using a full-wave simulator (HFSS). The meander combline structure was fabricated using conventional PCB technology. The thickness of the FR-4 laminate was $730 \mu \mathrm{m}$ and low equivalent series resistance (ESR) surface mount capacitor were used to complete the filter structure. To characterize the filter two port network $S$-parameter measurements in $1-3 \mathrm{GHz}$ bandwidth were done. Very good agreement between the modeled and measured data was obtained (Fig. 8). The filter is centered at $2.38 \mathrm{GHz}$, and has a bandwidth of $110 \mathrm{MHz}$, with a minimum $2.3 \mathrm{~dB}$ attenuation.

\section{B. Embedded Transformer Balun}

The balun (balance to unbalance) is another important component of the front end circuitry. It has the function to change a single-ended signal, a signal that is referenced to ground, to a balanced signal with equal potential with respect to ground but opposite polarity. There are several configurations available to realize balun structure; one of the most widely used is Marchand configuration [21]. However, the main drawback of this configuration is the size of it, as it employs quarter wavelength transmission lines at operating frequency to create the desire phase shift between the signals. Another approach is to use a transformer to create the needed phase change [20]. Here, a multilayer transformer was embedded into the substrate reducing the area occupied by the balun. The key of this design is the combination between the inductance of the windings and capacitance 


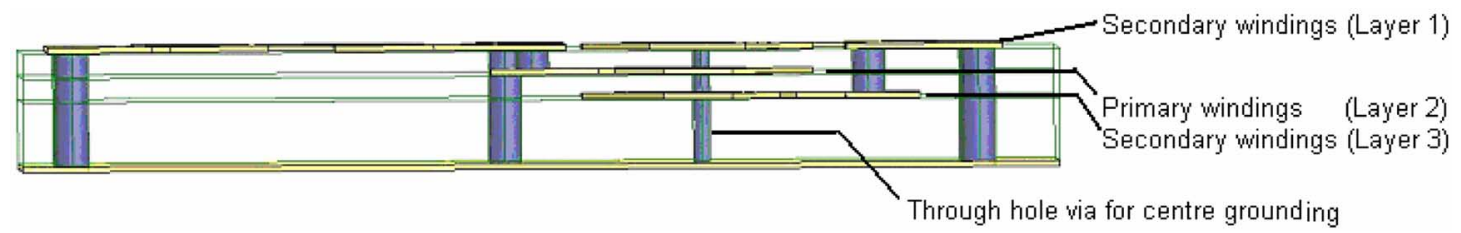

Fig. 10. Balun structure cross section.

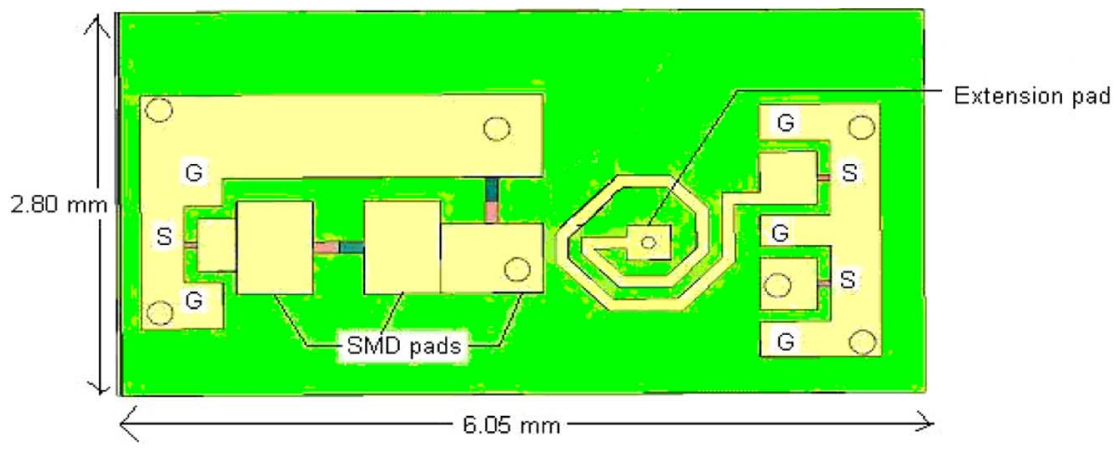

Fig. 11. Top view of the balun (layer 1).

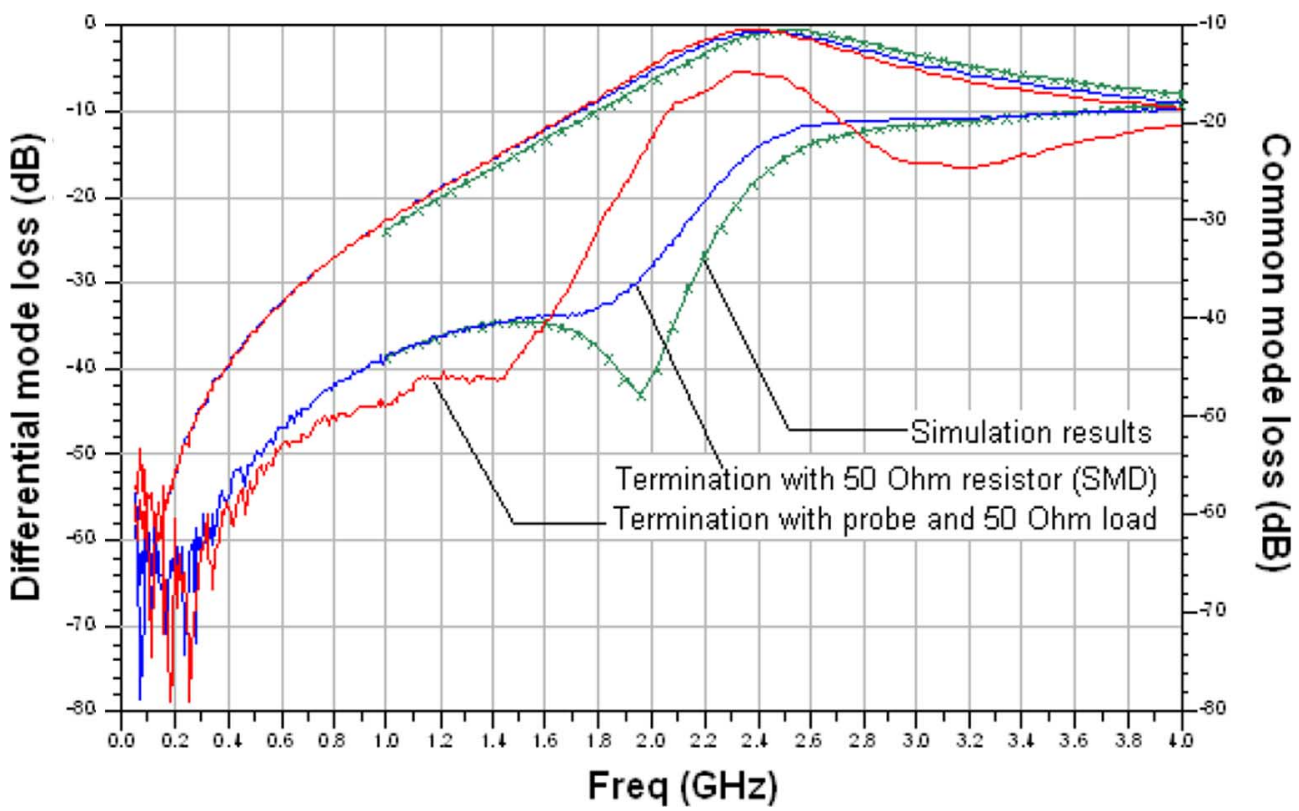

Fig. 12. Measured and simulated DML and CML for the balun.

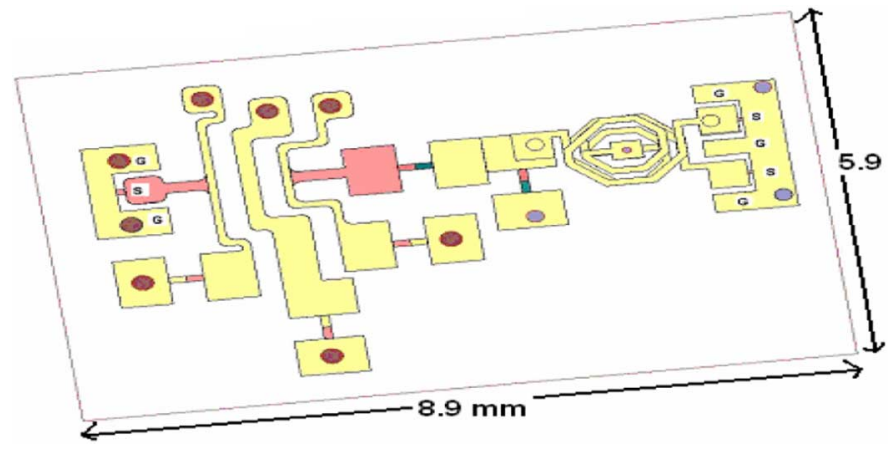

Fig. 13. Top view of the filter-balun 3-D model. of an external capacitor to achieve the required impedance transformation and phase shift. The balun for the Bluetooth system needs to create an impedance transformation from $50 \Omega$ unbalance to $100 \Omega$ balance impedance needed at the input of the RF chip. The balun circuit model is shown in Fig. 9, where port one is single ended, and port two and three are the differential ports. Two capacitors are used for matching the single ended input to the load impedance. The balun coils were design in octagon shapes and were distributed over three layers. The primary coil is on layer two and the secondary coil is distributed over the layer one and three. This 3-D design (Fig. 10) enables a more compact structure with better coupling. The necessary grounding is done through a via located at the inner end of the 


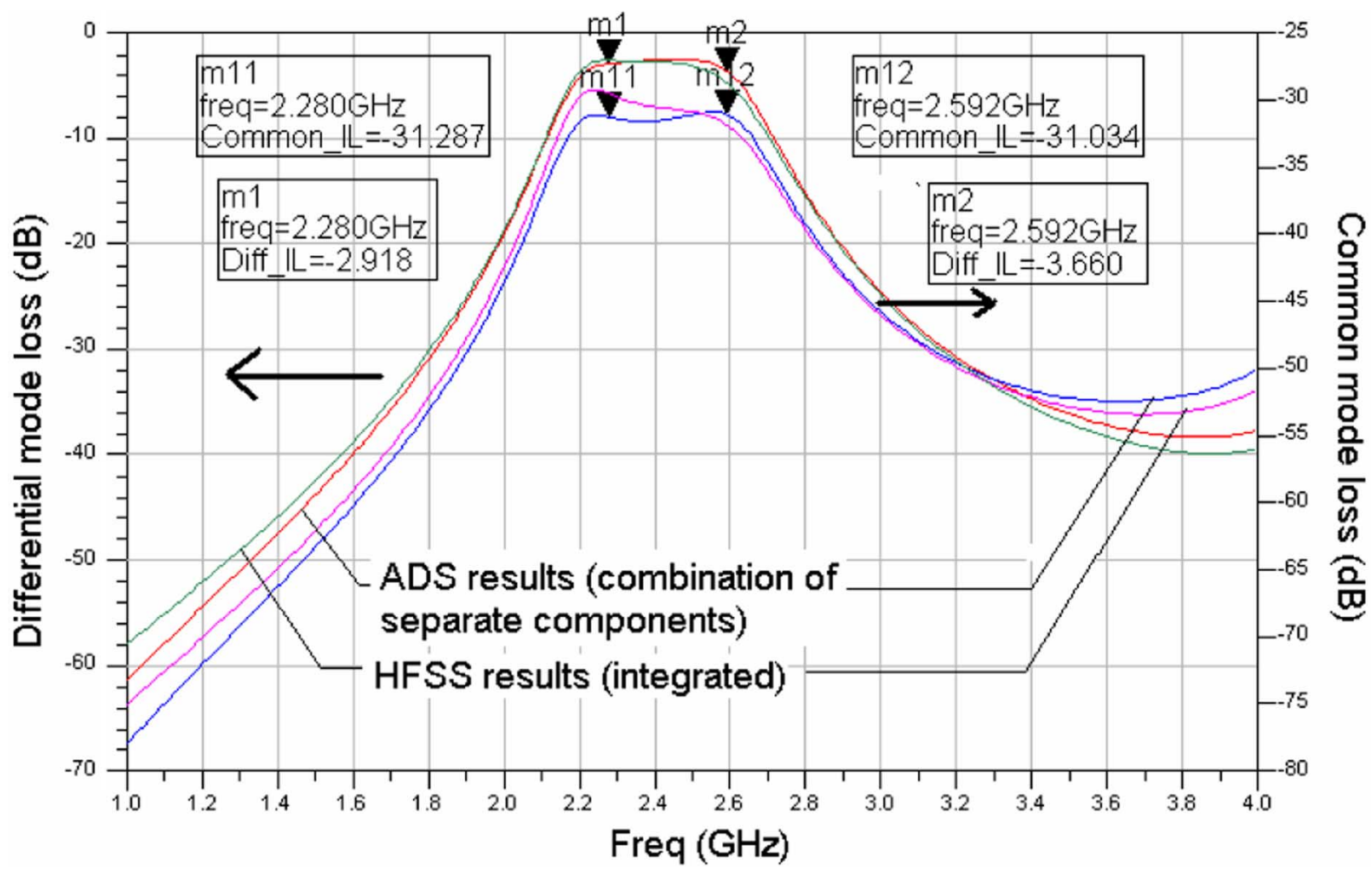

Fig. 14. DML and CML fro the filter-balun chain—full-wave model circuit model comparison.

windings, and connected to the bottom ground on layer four (Figs. 10 and 11).

The above structure was solved and optimized using HFSS. The balun was fabricated using regular PCB technology and measured using a two port measurement technique. Due to the fact that the balun is a three port device and the available vector network analyzer (VNA) was only two port, three measurements are needed to fully characterize the device. Each measurement is a two port measurement with the third unused port terminated with $50-\Omega$ broadband termination. Initially the termination of the unused port was done through a FPC GSG $400-\mu \mathrm{m}$ Cascade probe on which a broad band $50-\Omega$ termination was attached through the coaxial port. Measurements were done in the 100-MHz to 4-GHz bandwidth and the differential mode insertion loss (DML), common mode insertion loss (CML), and amplitude imbalance for the balun were extracted. The results of the common and differential loss are shown in Fig. 12. One can notice that the DML matches very well with the simulation results but for the CML the discrepancies between the measured and simulated data are quite large. The DML and CML are computed using (10) and (11). It can be notice that for the differential case any error that might have been introduced through the measurement process is subtracted out as long as is consistent (same error with the same value for all the measured cases). However, for the CML case the error adds-up and the results are very different than expected

$$
\begin{aligned}
& \mathrm{DML}=20 \times \log 10\left(\frac{|S 21-S 31|}{\sqrt{2}}\right) \\
& \mathrm{CML}=20 \times \log 10\left(\frac{|S 21+S 31|}{\sqrt{2}}\right) .
\end{aligned}
$$

Following this observation the termination using the probe with the $50-\Omega$ load was changed with a $50-\Omega$ solder resistor, and the measurements were repeated. The results showed much better agreement with the simulation data (Fig. 12). The error noticed in the first instance was due to the longer return path for the current due to the FPC probe, which was added to the measurement set up but was not calibrated out.

Low ESR 0402 surface mount 0.5 -pF capacitors were used on the measured test vehicle. With this setup a $290-\mathrm{MHz}$ bandwidth with amplitude of $-2 \mathrm{~dB}$ and phase imbalance of $7^{\circ}$ was obtained. However, a shift in the designed center frequency was noticed, instead of $2.45-\mathrm{GHz}$ center frequency a $0.2 \mathrm{GHz}$ shift to $2.25 \mathrm{GHz}$ was obtained. Further investigation through correlation of the simulation and measurement results showed that the shift is mainly because of an extra capacitive parsitics present in the fabricated structure due to a thinner dielectric layer in the fabricated test vehicles. In order to compensate for this shift the input capacitance was reduce to $0.4 \mathrm{pF}$. The new setup had a smaller bandwidth of $190 \mathrm{MHz}$ where the amplitude imbalance was better than $-2 \mathrm{~dB}$ and the center frequency has been shift to $2.42 \mathrm{GHz}$, hence the Bluetooth ISM band is well covered. By using the $50-\Omega$ solder resistor as termination for the unused port, the phase imbalance was improved to within $4^{\circ}$.

\section{Filter-Balun Integration}

The proximity of the filter to the balun and their distributed characteristics can create unwanted electromagnetic coupling between the two embedded structures. To make sure that parasitic coupling between them is minimized a simple simulation experiment was set up. The full chain (Fig. 13) filter and balun was model in the full-wave simulation environment. The 


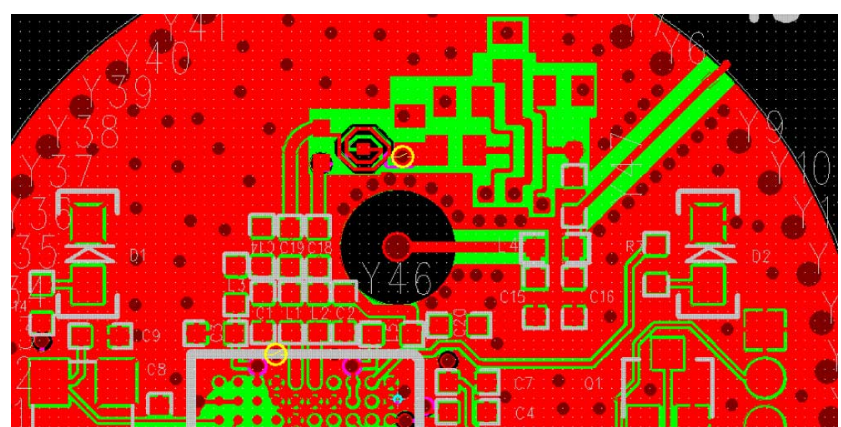

Fig. 15. Layout of the sixth layer-the filter, balun, and via connection to the antenna feed are visible.

HFSS 3-D model was able to capture any possible electromagnetic field coupling between the two devices. Separately HFSS models of the balun and filter only were solved and the $S$-parameter of each block was imported in a circuit solver.

The chain was then built, using the $S$-parameter data into the circuit simulator (ADS from Agilent Technologies, Santa Clara, CA) and solved. In this model no coupling was included. The results were compared in terms of amplitude and phase. As the chained structure was a three port device, a DML and CML were computed to use the result as comparison gauge (Fig. 14). The $S$-parameter results were also compared. Good agreement was obtained between the circuit model and full-wave solution was obtained for the case shown in Fig. 13. This gave us confidence that the coupling between devices is minimized for this particular arrangement of the filter and balun and will not interfere with a normal functionality of the system. This arrangement (Fig. 13) was used in the final module (Section IV).

\section{SIP INTEGRATION AND FABRIACTION}

As mentioned earlier the integration platform used was regular PCB substrate on which all the above described blocks, antenna, filter, and balun could be integrated together with the rest of the active components to form a completely functional Bluetooth system.

The module substrate had six layers. The first top two layers were used for the antenna feed layer. The third layer was used as a general grounding for the whole module. The fourth and fifth layers are for the power and ground necessary for the active ICs which are mounted on the sixth layer. The fourth, fifth and sixth layers were also used to integrate the transformer balun and the combline filter. The magnetic dipole located on the top layer used to feed the antenna was connected to the bottom layer through $50 \Omega$ characteristic impedance structured formed by an inner via and surrounding grounding via, as shown in Figs. 15 and 16 . The grounding vias connects the ground from the sixth layer to second layer which is the antenna ground.

The active components, the Bluetooth SOC (BC04-ROM, from CSR), the voltage controller, the crystal oscillator, the extra RAM, and all the other passive components were surface mounted on the sixth layer of the module. The module also had a USB port to provide the necessary power and data transfer. Three types of module were design and fabricated using the same size and shape of the substrate. First module has used

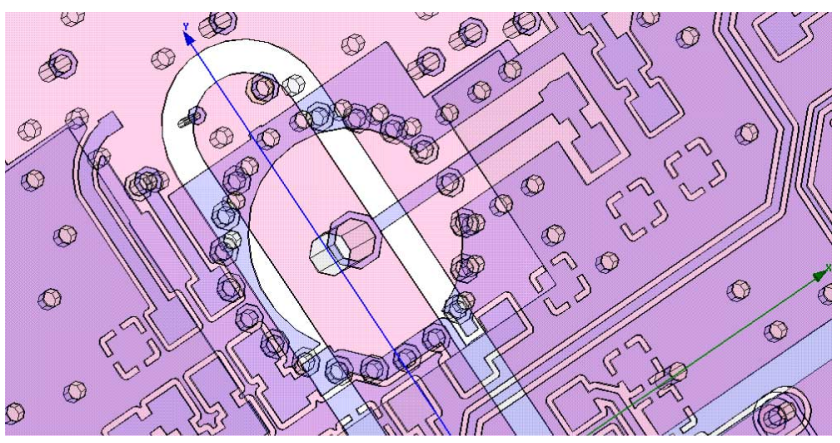

Fig. 16. $50 \Omega$ through via connection to the antenna feed (3-D model).

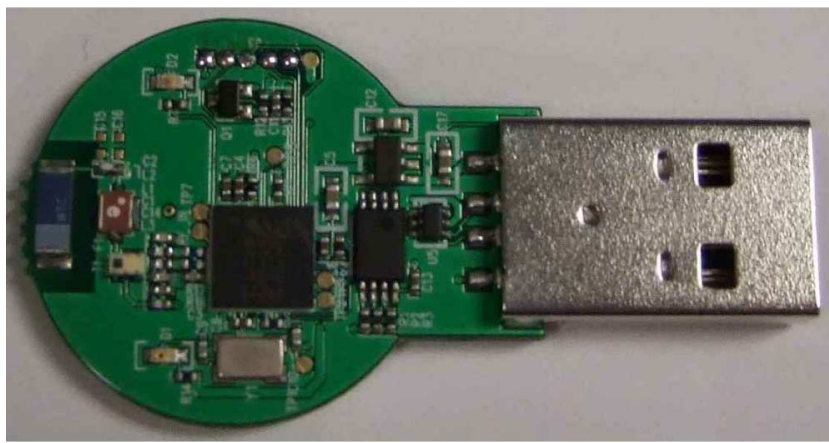

Fig. 17. "Classical” Bluetooth module test vehicle (test veh1).

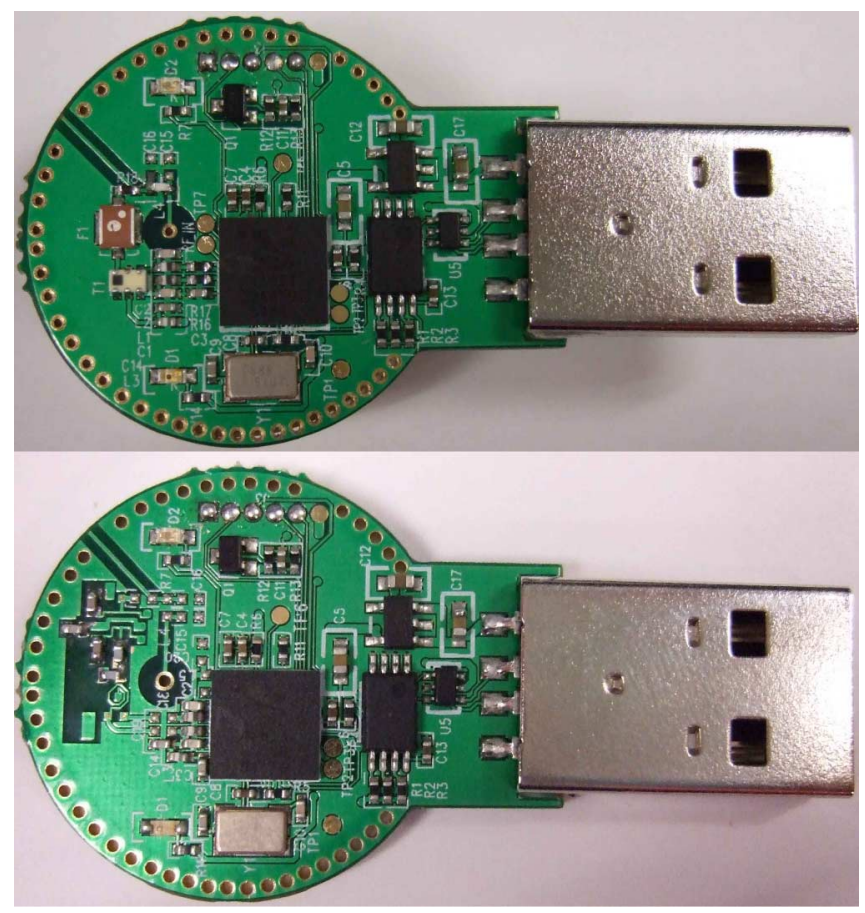

Fig. 18. Bottom side view of test veh2 top and test veh3 bottom-test veh3 has the embedded front end (filter and balun).

exclusively commercial available components including the antenna the filter and the balun in the front end [4], [5], [22], [23] (Fig. 17). The second test module used the dielectric resonator package integrated antenna but the rest of the module was the same as in the first case (commercially available filter and balun) (Figs. 18 and 19). The third module design has used 


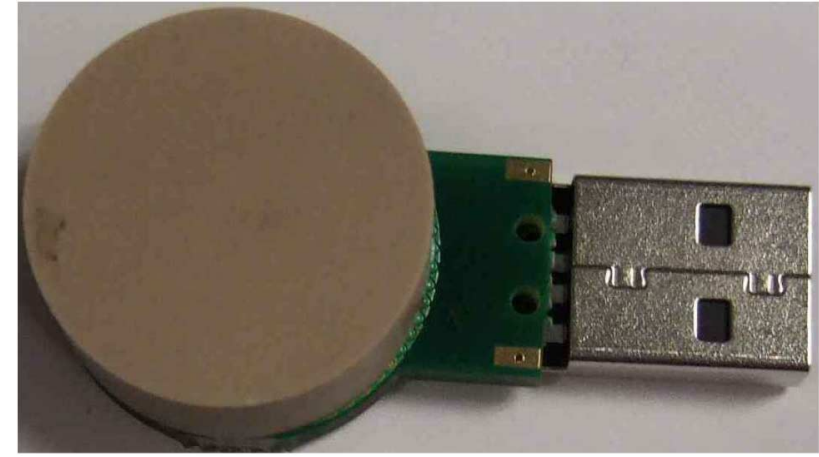

Fig. 19. Top side view of test veh3.

the embedded front end described in Section III and the antenna described in Section II (Figs. 18 and 19).

One complication brought in by the new balun is the way in which the direct current (dc) supply to bias the Bluetooth is implemented. Extra blocking capacitors are needed it to be added into the input of the chip to separate the dc path from the $\mathrm{RF}$ path, other wise the ground of the system can get corrupted by the dc bias due to the common grounding via used into the balun to short all the three coils to the ground. The dc bias of 1.8 $\mathrm{V}$ [4], [5] needed it to the RF input has to be provided to both of the input ports. This will add an extra surface mount capacitor and inductor for the biasing network.

\section{Functional Testing}

The test vehicles described in Section IV were functional tested by using them in a simple experiment, in which a Bluetooth enable phone was used to send and receive files to a laptop on which the new module was connected through the USB port. The laptop with the module was used to send a file to the phone. The distance between the base (laptop + Bluetooth module) and the receiver (Bluetooth enable phone) was increased by moving the phone further and further. Both the Bluetooth module integrated into the mobile phone and our implementation were class II with a maximum output power of $2.5 \mathrm{~mW}(4 \mathrm{dBm})$, which have a nominal range up to $10 \mathrm{~m}$, for transmitter and it has a sensitivity of $-70 \mathrm{dBm}$ (BER $0.1 \%$ ) for the receiver. The experiments described in this section were done in two environments, a long corridor where a direct site with no blocking between the two devices was available and an office environment where many obstacles were present (cubicle walls, desks, etc.).

The first step was to pair up the two Bluetooth devices, once paired-up the information can be sent between them. The "classic" module (test veh1) that has all the commercial available components (Fig. 17) was used first for the experiment. Once paired the phone was positioned one meter away from the laptop on which the Bluetooth was installed and we try to connect to the phone by sending a picture file.

The connection was done without any problems and we canceled the file transfer and the phone was moved further and the procedure repeated. No connection problem was noted until a distance of approximate $8-10 \mathrm{~m}$ was reached. In this range a difficulty in connecting was noticed. But most of the times when the connection was tried it was successful. Further than $10 \mathrm{~m}$ it

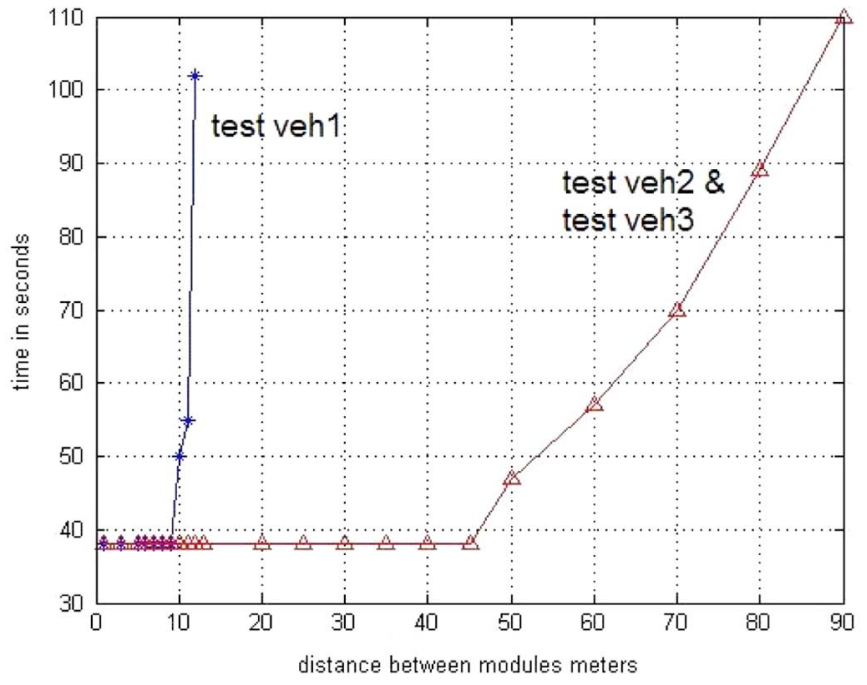

Fig. 20. Time ( $y$ axis)/Distance ( $x$ axis) variation for the three test vehicles.

was more and more difficult to connect and most of the times an error message of unable to make the connection was displayed by the software managing the wireless connection. It has to be mentioned that we were able to connect the two modules up to $15-17 \mathrm{~m}$ but only in certain case.

The same experiment was repeated with the other two modules on with the packaged DRA and the other one with the DRA and embedded front end (test veh2 and test veh3). Both yield similar results. The new module was able to connect to the Bluetooth phone without any problems up to $60-65 \mathrm{~m}$. After $70 \mathrm{~m}$ we found that is more difficult to connect the two devices but connection was established up to $90 \mathrm{~m}$. As mentioned before both of the two test vehicles had similar performances and we have attributed the boost in the performance to the DRA and good RF design.

The second experiment that was to send a file via the wireless connection and to move the receiver while the file is being sent further and further until the connection is broken. With first test vehicle the connection was broken around 16-20 m range. For the second and third test vehicle the range was extended all the way to $100-120 \mathrm{~m}$.

In our third experiment we have recorded the time required for the data transfer. In a typical test case, we observed how long it takes for a $1500 \mathrm{kB}$ JPEG picture file to be transferred between the laptop to the Bluetooth enabled phone through the wireless connection. Using the first test vehicle the file transfer was done in about $38 \mathrm{~s}$ when the distance between the two devices was less than $9 \mathrm{~m}$. At $10 \mathrm{~m}$ away it took about $50 \mathrm{~s}$ to transfer the same file and at $12 \mathrm{~m}$ it took $102 \mathrm{~s}$. For distances larger than $12 \mathrm{~m}$ the full file transfer had failed to take place. When the second and third test vehicles were used the distance at which $1500 \mathrm{kB}$ file can be transferred in $38 \mathrm{~s}$ was extended up $45 \mathrm{~m}$, and it took about $47 \mathrm{~s}$ at $50 \mathrm{~m}$ and at $60 \mathrm{~m}$ distance it was $57 \mathrm{~s}$. The maximum distance was about $100 \mathrm{~m}$ at which the measured time was $110 \mathrm{~s}$ for the file to be transferred. The results of this experiment are summarized in Fig. 20.

The experimental results characterized the overall module performance in term of data transfer rate. The speed of data 
transmission depends on the bit error rate of the communication channel. For the bit error rate below a definite threshold the Bluetooth operates at maximum data rate, which is in our case of about $40 \mathrm{~kb} / \mathrm{s}$. When the bit error rate is above the threshold and the transmitted data is severely corrupted, the Bluetooth hardware requests retransmission of the corrupted data until an acknowledgement is received indicating a successful transmission (or until a predefined time-out occurs). Since retransmission of the corrupted data requires additional time, the overall data rate through the channel will be reduced as the channel throughput is reduced. On the other hand, the bit error rate depends on signal to noise ratio at the receiver output. Therefore, the results presented in Fig. 20 indicates that the signal to noise ratio at the receiver output for the test vehicles 2 and 3 is maintained above the threshold level up to the distance of $45 \mathrm{~m}$, while the for the standard Bluetooth module that distance was limited up to $9 \mathrm{~m}$.

The performance degradation of the test vehicles 2 and 3 with the distance was less abrupt compared to the test vehicle 1 . We believe that these improved performances observed for the test vehicle 3 and 2 are mainly due to the good RF design and superb characteristics of the DRA and the design methodology employed (SieP). The reason most wireless modules lack range is due to their poor RF design, poor matching can block most of RF power from and out of the chip. A lesser known and often ignored problem is detuning of antenna. Most of the antennae are designed to have maximum at the center of their frequency band, as they become smaller they become more dependent of the surrounding ground plane hence prone to severe detuning, shifting the maximum gain to a different frequency than desired. In this case the packaged integrated DRA is optimized for the size and the shape of the existent ground plane and due to its working principle and inherent property, it cannot be easily detuned, as in case of the small chip antenna used in test vehicle $1[23]$.

\section{CONCLUSION}

Existent and emerging wireless devices need integration platforms that can take care of a diversity of technologies that can fulfill the required functional and economical needs. SoC may not be best solution for some RF systems, depending on the required functions, specifications, time, or target cost. However, conventional board level solution may not be acceptable either because of its large size, high-power consumption, and high cost. The SieP presented here using FR4 substrate with a package dielectric resonator antenna can provide high performance, low-power consumption at a lower cost. The excellent performance of the module is attributed to the DRA and the novel integration methodology ( $\mathrm{SieP}$ ) employed in this work. The work done in this project has demonstrated a way to interface and integrate an excellent antenna with the rest of commercial available technologies to realize a high performance functional module. Further integration for miniaturization embedding the front end-filter and balun - has also been demonstrated. The effectiveness of the new module was measured against a similar module in which conventional technologies employing - chip antenna and surface mount front end components were used to realize the module. Improvements in wireless link coverage area and power consumption have been obtained, demonstrating the efficiency of the new concept of integrated package antenna module. However, the present Bluetooth system standard demonstrated here may not be the most stringent and may not demand such a wide band and high efficiency antenna. At same time the integrated solution is highly inspiring and capable of supporting the upcoming enhanced data rate Bluetooth standards. Further more the design concept and methodology demonstrated in here has higher potential to cater to more demanding wireless access technologies such as MOFDM UWB or WLAN.

\section{REFERENCES}

[1] Prismark Wireless Technology Report Prismark, Cold Spring Harbor, NY, 2006.

[2] R. Tummala, "Moore's law meets its match," IEEE Spectr., vol. 43, no. 6, pp. 44-49, Jun. 2006.

[3] P. Wambacq et al., "Chip-package co-design of a $5 \mathrm{GHz}$ RF front-end for WLAN," in Int. Solid-State Circuits Conf. Tech. Dig., Feb. 2000, pp. 318-319.

[4] DEV-PC-1487 BlueCore4-External Class 2 Example Design Cambridge Silicon Research, Camrbidge, U.K. [Online]. Available: http://www.csr.com

[5] DEV-PC-1583A Class 2 Low Cost USB Dongle Example Design Cambridge Silicon Research, 2006, Cambridge Silicon Research. Cambridge, U.K. [Online]. Available: http://www.csr.com,

[6] S. Brebels et al., "SOP integration and codesign of antennas," IEEE Trans. Adv. Packag., vol. 27, no. 2, pp. 341-351, May 2004.

[7] A. P. Popov and M. D. Rotaru, "A novel integrated dielectric resonator antenna for circular polarization," in Proc. 53rd Electron. Compon. Technol. Conf., May 27-30, 2003, pp. 470-473.

[8] C.-P. Chua, P. A. Pavlovich, and M.-S. Leong, "Design and optimization of integrated dielectric resonator antennas for bluetooth applications," presented at the ISAP, Sendai, Japan, 2004.

[9] C.-P. Chua, P. A. Pavlovich, and M.-S. Leong, "A compact and wideband rectangular dielectric resonator antenna," in 8th Electron. Packag. Technol. Conf., Singapore, Dec. 2005, vol. 1, pp. 313-316.

[10] J. Laskar et al., "Development of integrated 3-D radio front-end system-on-package (SOP)," in Tech. Digest 23rd Annu. GaAs IC Symp., Oct. 2001, pp. 215-218.

[11] K. Lim, A. Obatoyinbo, M. Davis, J. Laskar, and R. Tumamal, "Development of planar antennas in multi-layer packages for RF system-on-apackage applications," Proc. Electrical Performance Electron. Packag. , pp. 101-104, Oct. 2001.

[12] D. Heberling, "Modern trends in the development of small and handy antennas," in Proc. 2001 Microw. Optoelectron. Conf., Belem, Brazil, Aug. 6-10, 2001, vol. 2, pp. 59-64.

[13] Y. P. Zhang, "Integration of microstrip antenna on cavity-down ceramic ball grid array package," Elerctron. Lett., vol. 38, no. 22, pp. 1307-1308, Oct. 2002.

[14] P. C. T. Song, P. S. Hall, and H. Ghafouri-Shiraz, "Novel RF front-end antenna package," IEE Proc. Microw., Antennas, Propagat., vol. 150, pp. 290-294, Aug. 2003.

[15] J. Ryckaert et al., "Single-package $5 \mathrm{GHz}$ WLAN RF module with embedded patch antenna and $20 \mathrm{dBm}$ power amplifier," in Microwave Symp. Dig. IEEE MTT-S Int. Symp. IMS, Jun. 2003, vol. 2, pp. 8-13.

[16] R. L. Li, G. Dejean, M. M. Tentzeris, and J. Laskar, "Development and analysis of a folded shorted-patch antenna with reduced size," IEEE Trans. Antennas Propag., vol. 52, no. 2, pp. 555-562, Feb. 2004.

[17] K. Lu-Wong, Planar Antennas for Wireless Communication. New York: Willey, 2003.

[18] L. Y. Ying and M. D. Rotaru, "A compact integrated ISM bandpass filter based on combline structures on organic substrate," in Electron. Syst.-Integration Technol. Conf., Sep. 2006, pp. 802-804.

[19] S. Caspi and J. Adelman, "Design of combline and interdigital filter with tapped-line input," IEEE Trans Microwave Theory Tech., vol. 36, no. 4, pp. 759-763, Apr. 1988.

[20] Y. J. Yoon et al., "Spiral transmission line baluns for RF multichip module packages," IEEE Trans. Adv. Packag., vol. 22, no. 3, pp. 332-333, Aug. 1999.

[21] H. Y. D. Yang and J. Castaneda, "Design and analysis of on-chip symmetric parallel plate coupled-line balun for silicon RF integrated circuits," in IEEE Radio Frequency Integrated Circ. Symp., 2003, pp. $527-530$. 
[22] Murata Microwave Components. p. 327, Specification for chip multilayer hybrid baluns.

[23] Yageo Passive Components Data Sheet-3216 Ceramic Chip Antenna for Bluetooth/WLAN Applications.

Rotaru Mihai (M’00) was born in Cluj-Napoca, Romania, on May 141973. $\mathrm{He}$ received the B.Eng. and M.Sc. degrees from Technical University of Cluj, Cluj, Romania, in 1996 and 1997 respectively, and the Ph.D. degree in electrical engineering from University of Southampton, Southampton, U.K., in 2000

From 2000 to 2001, he was a Research Assistant with the School of Engineering and Mathematical Sciences, City University London, London, U.K. Between 2001 and 2007, he was member of technical staff with Microsystems, Modules and Components Laboratory at Institute of Microelectronics, Singapore. While in Singapore, he worked on design, simulation, and analysis of advanced packaging solution for microelectronics, SiP solutions for wireless applications, as well as electromagnetic modeling of 3-D stack modules. In June 2007, he joined the School of Electronics and Computing Science at University of Southampton, Southampton, U.K. as a Lecturer. His research interests include efficient numerical methods and modeling techniques for complex electromagnetic problems, dielectric resonator antennas, $\mathrm{SiP}$, and electromagnetic compatibility.

Dr. Rotaru is IET member. He has received the Best Poster Paper award at ECTC 2003. He was in the Technical Committee of IEEE EPTC 2004-2006. $\mathrm{He}$ is a member of IET Solent Network Committee.

Lim Ying Ying received the B.Eng. and M.Eng. degrees from Nanyang Technological University, Singapore, in 2003 and 2005, respectively.

She is currently with the Institute of Microelectronics, focusing on the design and measurement of embedded passives and RF interconnects. Her experience includes the fabrication of integrated passives on multilayer laminates, as well as characterizing for the dielectric properties of substrates and laminates across broadband frequencies.

Ms. Lim was the recipient of the Outstanding Student Paper Award at EPTC 2005.
Haridas Kuruveettil received the M.Tech. degree in digital communication engineering from Visweswaraiah Technological University (VTU) Bangalore, India, in 2001.

Since 2004, he has been with the Integrated Circuits and Systems Laboratory, Institute of Microelectronics, Singapore. He has been involved in the design of system in package solutions for wireless application as well as high-speed optoelectronic communication modules. At the moment, he is working on wireless system design with specialization in RF circuit design.

Yang Rui received the B.Eng. and M.Eng. degrees from Northwestern Polytechnical University, Xi' an Shaanxi, China. in 1996 and 1999. respectively. He is currently working toward the Ph.D. degree at Nanyang Technology University, Singapore.

From 2004 to 2007. he was a Research Engineer with Microsystems, Modules and Components Laboratory, Institute of Microelectronics, Singapore, working on design, modeling, and characterization of high-speed and high-frequency advanced packaging solutions. In July 2007, he joined Huawei Technology Company, Ltd., as Senior Hardware Engineer, where he is involved in board and package design/layout for signal integrity and power integrity for high-speed interconnects modeling and analysis.

Popov Alexander photograph and biography not available at the time of publication.

Chua Chee Parng photograph and biography not available at the time of publication. 